\title{
Mycose viscérale de Poissons dulçaquicoles tropicaux.
}

Note préliminaire

\author{
par G. CHAUVIER \\ (Collaboration technique : $\mathrm{M}^{\text {me }}$ J. Mortier-Gabet) \\ Ménagerie du Muséum national d'Histoire naturelle, \\ 57, rue Cuvier, F 75005 Paris.
}

\section{Résumé.}

Dans quatorze cas de mycose viscérale mortelle de poissons dulçaquicoles d'ornement, les lésions kystiques du foie et de la rate, ainsi que les manifestations cliniques de la maladie, ressemblent à celles de l'ichthyophonose. Le microorganisme responsable a pu être cultivé ; son identification à Ichthyophonus hoferi est discutée.

\section{Summary.}

\section{Visceral mycosis of tropical freshwater fishes.}

Fourteen cases of a systemic mycosis in ornamental fresh-water tropical fishes are recorded. Clinical signs, cystic lesions in the liver and spleen, recall those of an infection by the fungus Ichthyophonus, or Ichthyosporidium hoferi. The possibility that the fresh-water fishes' parasite might be of a different species is discussed. Morphological aspects of the yeast-like fungus in culture are described.

En 1976, au cours de l'examen nécropsique d'un Hemichromis bimaculatus, notre attention a été attirée par la présence dans le foie de très fines granulations, à peine visibles à l'œil nu. L'examen à la loupe montra qu'il s'agissait en réalité de formations kystiques enveloppées d'une membrane réactionnelle et d'une membrane propre enfermant un cytoplasme plurinucléé et très fortement chargé de granulations irrégulières de couleur brun-sombre, représentant probablement des substances de réserve. Les dimensions de ces kystes, isolés par microdissection et inclus dans le baume du Canada sont les suivantes : de $89 \times 76 \mu$ à $395 \times 345 \mu$.

Accepté le 5 octobre 1978. 
D'autres kystes furent placés en milieu aqueux stérile, entre lame et lamelle lutées à la paraffine, et furent observés pendant cinq jours en vue de déceler les toutes premières modalités de leur évolution. L'un d'eux émit dès le premier jour des éléments levuriformes ovoïdes uni ou bi-nucléés d'environ $5 \mu$ de grand axe et $2,5 \mu$ de petit axe. Les autres kystes donnèrent issue, entre le $2^{\circ}$ et le $5^{\circ}$ jour à des masses plasmodiales où secondairement, par fragmentation du cytoplasme, se formèrent des éléments levuriformes uni ou binucléés identiques aux précédents.

Ces faits, joints aux constatations antérieures, nous orientaient vers le diagnostic de mycose. Pour le préciser, nous avons tenté de cultiver le microorganisme d'une part en chambre humide, d'autre part dans des tubes renfermant de la gélose glucosée de Sabouraud, à 2 et $5 \%$ et gardés à la température du laboratoire. Les résultats ont été les suivants :

\section{I. - En chambre humide (fig. $l$ ).}

Dans la goutte de gélose placée en chambre humide, les kystes parasitaires ont évolué de deux façons différentes: soit en émettant tout près d'eux un « essaim»
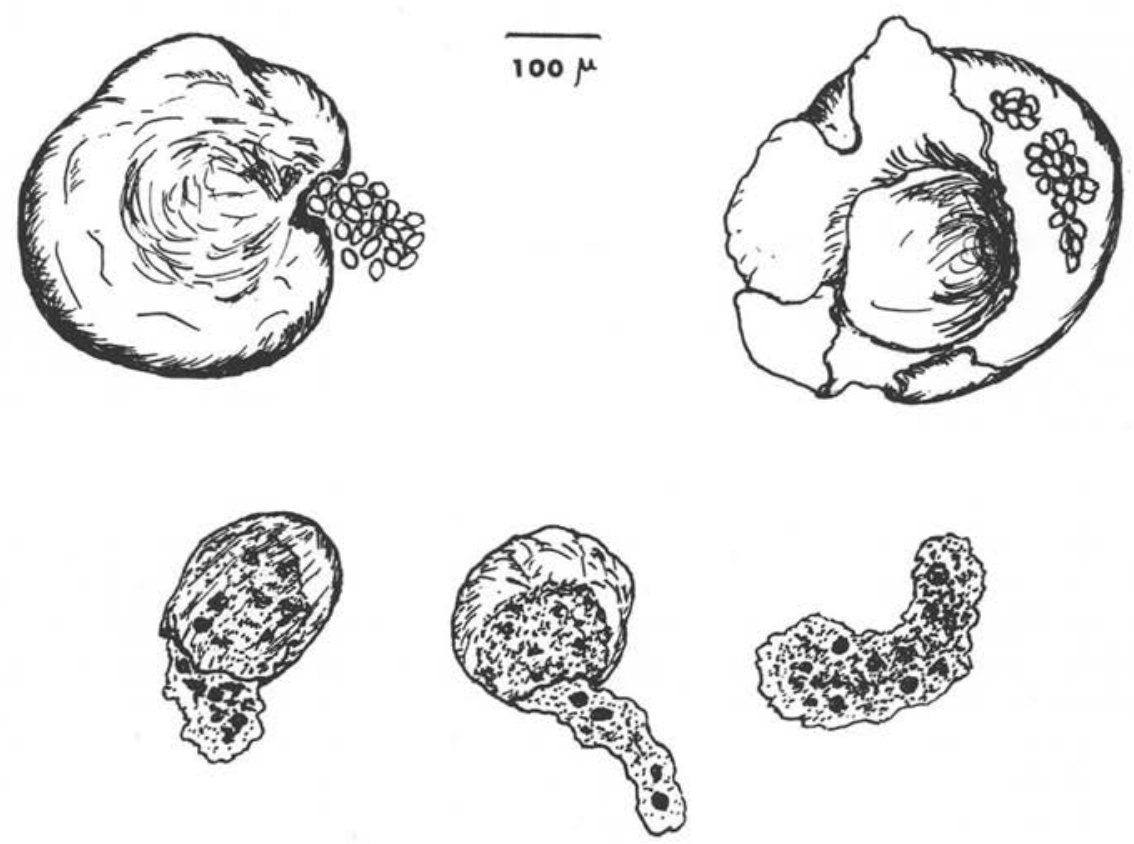

Fig. 1. En haut: déhiscence de gros kystes parasitaires en chambre humide; au-dessous : kystes moyens émettant des plasmodes, en milieu aqueux; près d'eux, un plasmode libre. 
d'éléments levuriformes plus ou moins agglutinés les uns aux autres et paraissant avoir été expulsés à travers une perforation de la membrane; soit en subissant une déchirure étoilée de cette membrane, avec repliement des lambeaux un peu à la façon de pétales. L'évolution paraissant s'être arrêtée à ce stade, même après le $5^{\circ}$ jour, nous avons préféré transférer ce matériel sur gélose en tubes, pour éviter de le perdre.

\section{II. - En cultures (fig. ?}

Les premières cultures, devenues bien visibles à la $48^{\circ}$ heure, se présentaient sous l'aspect de traînées blanchâtres, luisantes, crémeuses. Par la suite, en s'étendant à toute la surface de la gélose, elles prirent progressivement une teinte chamoisée. Le développement du microorganisme se fit aussi dans l'eau de condensation de la gélose, au fond du tube, en y amenant la formation d'un dépôt blanchâtre de plus en

\section{$\odot$}
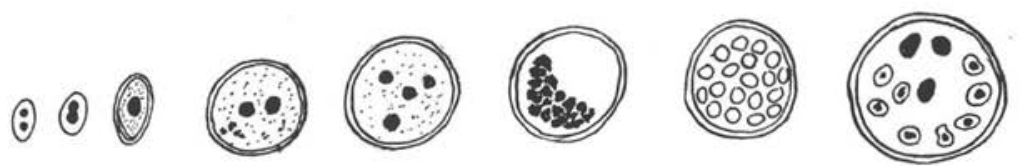

$10 \mu$
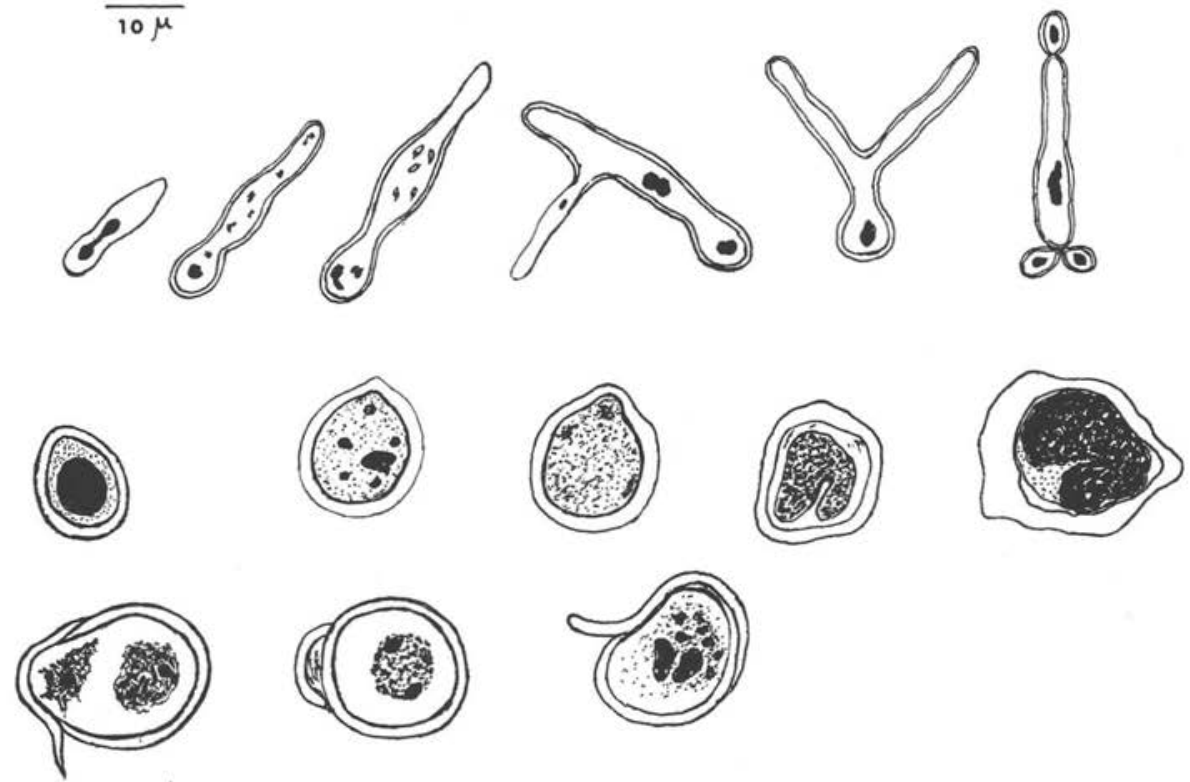

Fig. 2. Première rangée horizontale: éléments levuriformes ovoïdes, uni et binucléés, et évolution d'éléments levuriformes arrondis aboutissant à la production d'endospores. Deuxième rangée : ébauches mycéliennes. Troisième rangée : chlamydospore et quatre stades de formation de sporokystes. Quatrième rangée: trois aspects d'ookystes. 
plus abondant. Du point de vue microscopique, ces cultures se caractérisaient par la présence des diverses formes suivantes :

a) des éléments levuriformes ovoïdes, uni ou bi-nucléés, à membrane mince et cytoplasme homogène, réfringent, aux dimensions tout à fait comparables à celles des formes levures sorties des kystes parasitaires $(5 \mu \times 2,5 \mu)$;

b) des éléments levuriformes plus volumineux, arrondis, de $9,5 \mu$ de diamètre en moyenne, à membrane d'épaisseur variable, à cytoplasme granuleux renfermant une ou plusieurs masses nucléaires ;

c) des ébauches mycéliennes, se présentant sous l'aspect d'éléments allongés non cloisonnés ayant un peu l'aspect de «quilles» dont la partie renflée mesurait en moyenne $7 \mu$. de diamètre et se raccordait par un léger étranglement à un petit tube long de 25 à $30 \mu$; celui-ci légèrement renflé dans sa partie proximale, présentait ensuite une largeur de 4 à $5 \mu$ avec des bords à peu près parallèles, jusqu'à l'extrémité, arrondie ; celle-ci portait parfois un ou plusieurs bourgeons, ovoïdes, uninucléés semblables à certains des éléments levuriformes décrits en $a$ ) ; en quelques occasions, ces ébauches mycéliennes présentaient un début de ramification; dans leur cytoplasme, homogène le plus souvent, se trouvaient plusieurs petites masses nucléaires réfringentes, l'une d'elles étant en général située dans la portion renflée de la « quille».

\section{Autres cas}

Ils se sont manifestés sporadiquement au cours des années 1976, 1977 et 1978 (malgré les mesures de désinfection des aquariums appliquées après la mort de l'Hemichromis), sur trois autres poissons du même genre ainsi que sur cinq Cichlasoma biocellatum, trois scalaires et deux poissons rouges. Au début de chacune des cultures obtenues à partir des kystes prélevés sur ces poissons, nous avons constamment retrouvé les mêmes types d'éléments levuriformes et d'ébauches mycéliennes. Au cours des repiquages, les ébauches mycéliennes en forme de quilles ont paru devenir moins fréquentes, au fur et à mesure que les souches paraissaient s'adapter au milieu de culture ; les deux types d'éléments levuriformes étant, par contre, assez stables. Dans les cultures vieillissantes, sont apparues d'autres formes que nous nous bornerons à citer ici car leur description détaillée déborderait du cadre de cet article. Elles feront l'objet d'une autre note qui, nous l'espérons, permettra de jeter quelque lumière sur le cycle vital du parasite: c'étaient notamment des plasmodes, mais aussi des formes de résistance : chlamydospores, ookystes et sporokystes dans lesquels nous avons décelé la formation d'endospores. Il faut dire que, pour parvenir à établir le tableau complet du cycle végétatif du parasite in vitro, nous avons procédé minutieusement à une multitude d'examens réguliers de chaque culture de façon à la suivre longuement dans son évolution. Il peut être intéressant, à cet égard, de préciser que la longévité de nos cultures atteint maintenant vingt-quatre mois et que leur vitalité ne semble en rien diminuée, comme le prouvent les nombreux repiquages effectués aussi jusqu'ici. 
Il convient maintenant d'aborder le problème de la détermination de la position systématique de ce parasite fongique. Les premières recherches bibliographiques effectuées nous ont orientés vers le genre Ichthyophonus.

Les premiers stades d'évolution du parasite de nos poissons : déhiscence des kystes, fragmentation du cytoplasme des plasmodes, rappellent assez ceux que décrivent pour l'Ichthyophonus hoferi, Daniel, cité par Langeron, ainsi que Dorier et Degrange qui rappellent d'ailleurs que ces processus avaient déjà été observés par Hofer lui-même. Des similitudes existent aussi du point de vue symptomatologique. Avant de les envisager, un bref rappel historique permettra de mieux cerner le problème que pose cette infection, encore mal connue, plus de trois quarts de siècle après sa découverte.

C'est en effet en 1893 qu'elle fut observée pour la première fois par Hofer sur des Salmonidés. Un peu plus tard, Robertson l'observa sur des poissons marins (1908, 1909), de même que Johnstone (1906, 1913), Alexeieff (1914), Ellis (1930), Daniel (1933), Fish (1934), Sproston (1947).

Etudiant le parasite des poissons d'eau douce, Laveran et Pettit $(1910,1913)$ le transmettent non seulement de truite à truite, mais aussi à la perche et à la tanche. Plehn et Mulsow (1911), Neresheimer et Clodi (1914) tentent de préciser son évolution. Léger (1924) trouve un Ichthyophonus dans le tube digestif de la Lote d'eau douce et publie en 1927 et 1929 ses constatations sur les formes que revêt le parasite dans la truite. Dorier et Degrange $(1960,1961)$ étudient aussi son évolution chez la truite arc-en-ciel et le saumon de fontaine.

Des poissons tropicaux de divers genres entretenus en aquarium ont été reconnus atteints d'ichthyophonose par divers auteurs (Klemm et Freund, 1930 ; Lederer, 1936 ; Schäperclaus, 1939 ; Dyk, 1950 ; Wurmbach, 1951 ; Lewer, 1952 ; ReichenbachKlinke, 1954, 1956).

\section{Signes eliniques de l'infection.}

Dans les divers cas observés par nous, les signes cliniques concordaient bien avec ceux qu'indique Reichenbach-Klinke (à l'exception des lésions cutanées ulcéreuses). Ils se résument en : décoloration du tégument, précédée parfois par une phase d'assombrissement; amaigrissement de l'animal, se traduisant notamment par le fait que la convexité des masses musculaires bordant la nageoire dorsale s'atténue et peut même être remplacée par une légère concavité (ceci est aisément perceptible quand l'animal est vu de face) ; incurvation du corps d'un côté ou de l'autre ; incoordination des mouvements et troubles de l'équilibre allant parfois jusqu'au renversement à $180^{\circ}$, et à la nage sur le dos. Assez paradoxalement de tels troubles ne s'accompagnent pas toujours d'une diminution de l'appétit; dans l'un de nos cas, le poisson a nagé pendant quinze jours sur le dos sans cesser de s'alimenter. Une seule fois nous avons constaté une altération des nageoires, se traduisant par une perte partielle de substance au niveau de la nageoire caudale et par la disparition d'une nageoire pectorale, remplacée par un gros « bourgeon cicatriciel» de teinte rouge-vif. 


\section{Discussion}

Ce tableau clinique, les constatations nécropsiques, les premiers stades d'évolution des cultures, tendraient à étayer le diagnostic d'ichthyophonose. Mais il convient de noter tout de suite ici les remarques de Johnson et Sparrow (4) :

«... l'espèce $I$. hoferi n'a jamais été formellement circonscrite ; ... l'impression qui se dégage de la lecture des publications est que le champignon est extrêmement polymorphe et ceci fait qu'il est éminemment possible que l'Ichthyophonus hoferi ne soit pas une espèce unique ».

Inversement, Reichenbach-Klinke, dans son ouvrage sur les maladies des Vertébrés inférieurs, en 1965, revient sur l'opinion qu'il exprimait onze ans plus tôt (en même temps que Schäperclaus) quant à la possibilité de l'existence d'une espèce d'Ichthyophonus propre aux poissons tropicaux d'eau douce. Il estime que les différences entre les formes marines et les formes d'eau douce du parasite sont des conséquences de l'action de divers facteurs du milieu, température et salinité notamment.

Il nous paraît important de constater le manque de concordance entre les descriptions de formes marines, faites par plusieurs des auteurs cités ci-dessus. Il paraît peu vraisemblable, en effet, que des différences de salinité et de température puissent, dans ce cas, suffire à expliquer ces discordances. Peut-être le pourraient-elles, par contre, dans le cas des truites d'élevage chez lesquelles l'ichthyophonose aurait souvent pour origine la consommation d'aliments composés industriels contenant de la farine de poissons de mer infectés. Disposant maintenant, grâce au parasite des poissons tropicaux d'eau douce, d'un modèle expérimental bien défini, nous espérons apporter prochainement notre contribution à l'étude systématique du (ou des) parasite(s) jusqu'ici rassemblés dans le genre Ichthyophonus.

\section{Conclusion}

Qu'il s'agisse d'ichthyophonose proprement dite, à $I$. hoferi, ou d'une infection provoquée par un germe voisin, la mycose viscérale mortelle que nous avons décelée chez les poissons tropicaux dulçaquicoles est intéressante à plus d'un titre.

Du point de vue de l'aquariologiste, elle constitue une menace grave pour sa collection, en raison des risques de contagion et des difficultés de désinfection et de traitement. Du point de vue économique, lorsqu'il s'agit d'aquaculture, en eau douce ou même en eau de mer, l'ichthyophonose représente également un risque potentiel, non négligeable, de pertes importantes.

Enfin, du point de vue sanitaire et médical, il n'est pas inutile de rappeler qu'Euzéby signale la possibilité de troubles digestifs graves chez l'homme, après consommation de poissons atteints d'ichthyophonose. 


\section{Bibliographie}

1. Dorier (A.), Degrange (Ch.), 1960-61: L'évolution de l'Ichthyosporidium (Ichthyophonus) hoferi (Plehn et Mulsow) chez les Salmonides d'élevage (Truite arc-en-ciel et Saumon de fontaine). Trav. Lab. Piscicult. (Univ. Grenoble), 52-53, 7-44.

2. Euzéby (I.), 1969 : Cours de Mycologie médicale comparée, Vigot, édit., Paris, 330 p.

3. Fijan (N.), Maran (B.), 1976: Slucaj Ihtiosporidioze u pastrva. Veterinarski Archiv, 46, 65-67.

4. Johnson (T.W.Jr.), Sparrow (F.K.Jr.), 1961 : Fungi in Oceans and Estuaries, Hafner Publ. Cy, New York.

5. Langeron (M.), 1945 : Précis de Mycologie, Masson, édit., Paris, 674 p.

6. Laveran (A.), Pettit (A.), 1910: Sur une épizootie des truites, C.R. Acad. Sci., 151, 421-423.

7. Léger (L.), Hesse (E.), 1923 : Sur un champignon du type Ichthyophonus parasite de l'intestin de la truite. C.R. Acad. Sci., 176, 420-422.

8. Pettit (A.), 1913: Observations sur l'Ichthyosporidium et sur la maladie qu'il provoque chez la Truite. Ann. Inst. Pasteur, 27, 989-1008.

9. Reichenbach-Klinke (H.), Elkan (E.), 1965: The principal diseases of lower vertebrates, Academic Press., édit., New York, Londres, 600 p.

10. Sartory (A.), 1920: Champignons parasites de l'homme et des animaux, 895 p.; supplément: 78 p., Arsant, édit., St-Nicolas-du-Port. 